\title{
On fully stressed design and p-norm measures in structural optimization
}

\section{Zhou, Mingdong; Sigmund, Ole}

\section{Published in:}

Structural and Multidisciplinary Optimization

Link to article, DOI:

$10.1007 / \mathrm{s} 00158-017-1731-3$

Publication date:

2017

Document Version

Peer reviewed version

Link back to DTU Orbit

Citation (APA):

Zhou, M., \& Sigmund, O. (2017). On fully stressed design and p-norm measures in structural optimization. Structural and Multidisciplinary Optimization, 56(3), 731-736. https://doi.org/10.1007/s00158-017-1731-3

\section{General rights}

Copyright and moral rights for the publications made accessible in the public portal are retained by the authors and/or other copyright owners and it is a condition of accessing publications that users recognise and abide by the legal requirements associated with these rights.

- Users may download and print one copy of any publication from the public portal for the purpose of private study or research.

- You may not further distribute the material or use it for any profit-making activity or commercial gain

- You may freely distribute the URL identifying the publication in the public portal

If you believe that this document breaches copyright please contact us providing details, and we will remove access to the work immediately and investigate your claim. 


\title{
On fully stressed design and P-norm measures in structural optimization
}

\author{
Mingdong Zhou and Ole Sigmund $\bowtie$
}

Received: date / Accepted: date

\begin{abstract}
This brief note revisits the fully stressed design schemes and P-norm measures used in stress-based structural optimization. Two simple shape optimization cases are used to remind the reader that fully stressed designs only are optimal when unimpeded by geometrical restrictions and that high values of the stress norm are needed in order to achieve satisfactory designs.
\end{abstract}

Keywords Structural shape optimization · Stress minimization · Fully stressed design · P-norm constraints

\section{Introduction}

Achieving a fully stressed design (FSD) has been a driver in structural optimization from its beginning. A FSD state is reached when all structural members experience the same stress level. A myriad of structural optimization approaches have been based on achieving this constant stress state by simple update procedures that locally add material if the stress is high and subtract material if the stress is low, i.e. FSD techniques. Due to their simplicity and lack of requirements to additional sensitivity calculations, these approaches are sometimes also termed "non-gradient" or "gradient-less approaches".

Mingdong Zhou: Shanghai Key Laboratory of Digital Manufacture for Thin-walled Structures, School of Mechanical Engineering, Shanghai Jiao Tong University, No. 800 DongChuan Rd. Shanghai 200240, P.R. China.

E-mail: mdzhou@sjtu.edu.cn

Ole Sigmund: Department of Mechanical Engineering, Technical University of Denmark, Building 404, Nils Koppels Alle, DK-2800 Kgs. Lyngby, Denmark.

E-mail: sigmund@mek.dtu.dk
As long as a design is unrestricted by any geometrical constraints (or maximum local element densities or areas in topology optimization), the FSD is also the optimal design with respect to the minimization of maximum stress objective. Furthermore, the FSD is also optimal with respect to compliance, including $3 \mathrm{D}$, powerlaw non-linear elasticity and anisotropy (cf. ?). In shape optimization, where topology is fixed and only (parts of) outer boundaries may change, afore mentioned requirement is relaxed to hold only for all designed surfaces. That is, the tangential stress on all designed surfaces should take a constant value. Again, this criterion has been a driver for equi-stress, fully stressed or "gradient less" optimization formulations. And again we remind that this approach will yield optimal solutions both with respect to stress and stiffness design if the designed boundaries are geometrically unconstrained.

When studying the vast literature on structural optimization with stress constraints, it becomes clear that a very large fraction of test cases consider geometrically unconstrained problems. This probably explains the popularity of FSD approaches and may explain why even the International Organization of Standardization (ISO) has standardized the approach under the auspices of Biomimetics (?).

However, mechanical design problems are seldomly red geometrically unconstrained. Structural members cannot be arbitrarily thick or dense and typically, there is limited space and volumes reserved for other purposes in engine rooms. This results in complex and concavely shaped design domains. In this case, FSD approaches result in wrong and inefficient solutions. Although this deficiency of FSD should be well known and understood, it nevertheless seems to be ignored in many works - as an extreme case one may think about above mentioned ISO Standard from 2015. 
Admittedly, solving stress constrained structural optimization problems is hard and partially unresolved. Close to optimality, hundreds or even thousands of local stress constraints may become active (cf. ?), meaning that corresponding numbers of adjoint analysis must be performed and that design updates require advanced active-set strategies with many constraints. To partially circumvent this problem, many works use constraintaggregation strategies, for example by minimizing the $p$-norm of the stress instead of the maximum stress (cf. ??). When $p$ goes to infinity, the original objective is recovered, however, the optimization problem becomes exceedingly unstable. Therefore, many works use rather low $p$ values (down to $p=2$ ) which may result in designs that do not satisfy the original stress objective at all. ${ }^{1}$

In this brief note we (re-)illustrate above discussion with two simple 2D shape optimization examples. Both are solved using our recently published shape optimization approach based on the Deformable Simplicial Complex (DSC) method (????). The structural geometries are parameterized with a second order triangular mesh and their shapes are updated by explicitly moving the structural boundary nodes in normal directions based on consistent adjoint sensitivity analysis. The designable nodes include all the boundary nodes in the design domain, while supporting nodes move tangential to the support plane only. Due to higher order elements and integration orders, no regularization or filtering is necessary for stable optimization, except for stress smoothing when evaluating the nodal stresses. The presented examples are limited to linear elasticity, one isotropic material and design independent loads, however, conclusions will be similar for more complex settings.

The remainder of this short note is organized as follows. Section 2 briefly introduces the optimization strategies for FSD and the $p$-norm measure. Two benchmark examples and associated discussions are given in Section 3. Conclusions are stated in Section 4.

\section{Shape optimization strategies}

\subsection{Fully stressed shape design approach}

The FSD algorithm for DSC is based on the idea of displacing the surface of the component in the direction of its normal according to the local magnitude of the stress. (???). Here, the goal is to achieve a uniform

\footnotetext{
1 In this note, we discuss the minimization of stress with a volume constraint. However, all conclusions hold for the dual problem of minimizing volume subject to stress constraint as well.
}

nodal von-Mises stress (FSD) $\sigma_{n}^{*}$ for all the designable nodes $\mathbf{z}$ on the structural boundary, while maintaining a fixed structural volume during the design process.

- Step 1: For each node $i$ on the designable boundary with coordinate $\mathbf{z}_{i}$, determine the nodal stress $\sigma_{n}\left(\mathbf{z}_{i}\right)$ by local averaging over its attached elements.

- Step 2: Apply a bi-section method to find a target nodal stress $\sigma_{n}^{*}$, such that:

$$
\begin{aligned}
\sum_{i} \max \left\{\min \left\{\tau \cdot\left(\frac{\sigma_{n}\left(\mathbf{z}_{i}\right)}{\sigma_{n}^{*}}-1\right), L_{i}^{\mathrm{upp}}\right\}, L_{i}^{\mathrm{low}}\right\} \cdot \nabla g_{i} \\
=V^{*}-V,
\end{aligned}
$$

where $\tau, L^{\text {upp }}$ and $L^{\text {low }}$ are the scaling parameter, the upper and lower move-limits, respectively, determined by the current node position ${ }^{2}$ and the extends of the design domain, $\nabla g$ is the shape gradient of the structural volume, $V^{*}$ is the initial structural volume and $V$ is the current structural volume;

- Step 3: Update the structural boundary nodes according to the following rule:

$$
\mathbf{z}_{i}^{\text {new }}=\mathbf{z}_{i}+\tau \cdot\left(\frac{\sigma_{n}\left(\mathbf{z}_{i}\right)}{\sigma_{n}^{*}}-1\right) \cdot \mathbf{n}_{i},
$$

where $\mathbf{n}_{i}$ is the normal vector of the node $i$;

- Repeat step 1-3 until stoping criterion is satisfied.

If the design goal is to achieve a predefined target stress level $\sigma^{*}$ over the structural boundary, the bisection scheme in Step 2 is bypassed and hence no volume control is imposed. For the numerical examples presented in Section 3 , the design process stops whenever the maximum nodal change between two consecutive iterations is less than $0.05 \%$ of the averaged element edge size.

\subsection{P-norm measure for consistent update}

The $p$-norm stress measure is defined as

$S=\left(\int_{\Omega} \sigma^{p}(\mathbf{x}) d \Omega\right)^{\frac{1}{p}} \approx\left(\sum_{j=1}^{N_{e}}\left(\sum_{k=1}^{M_{j}}\left(\sigma_{g_{k}}^{j}\right)^{p} J_{k} \omega_{k}\right)\right)^{\frac{1}{p}}$,

where $\Omega$ is the design domain, $N_{e}$ is the number of elements, $J$ and $w$ denote the element Jacobian and the weight for numerical integration, respectively, while $N_{e}$ and $M_{j}$ are the number of elements and Gaus points in element $j$, respectively.

With this $p$-norm measure, the update is based on adjoint sensitivity analysis and Mathematical Programming based design updates as detailed in ?. The adjoint approach is insignificantly more expensive compared to

${ }^{2}$ For numerical stability of the DSC method, each boundary node is only allowed to move within a certain range in each design iteration. 
the FSD approach since it simply requires the solution of one extra load case (the adjoint load given as the derivative of the P-norm stress measure with respect to the field variables).

\subsection{DSC implementation}

Shortly explained, shape updates are followed by efficient local mesh-updates (nodal repositioning and insertion of extra elements) in order to ensure high quality triangular meshes throughout the optimization history without a complete remeshing, even for large boundary movements. For further details on the DSC implementation, readers are referred to previous papers ????.

\section{Numerical examples}

This section shows two examples: 1) a simple, geometrically un-constrained cantilever beam case and 2) a much more challenging L-bracket case. The units are non-dimensionalized.

\subsection{Cantilever beam}

The design domain for the cantilever beam example is defined in Fig. 1(a), where the left edge is fixed and a distributed load of 3 is exerted over the right edge. The initial guess is shown in Fig. 1(b), which has a maximum element stress of $\sigma_{e}^{\max }=27.7$ and occupies $40 \%$ of the design domain.

Figs. 1(c-e) present the results of minimizing compliance, $p$-norm $(p=30)$ stress, and the FSD, respectively. All three problems result in the same optimized shape. The compliance values of the three designs are equal, as shown in Tab. 1. The element stress distributions are visually equal while the slight differences in maximum elemental stresses are partly due to local fluctuations and partly due to the $p$-norm case considering element stresses as the direct measure of optimality, whereas the FSD uses nodal averaged values as driver. Furthermore, the DSC mesh is changing throughout the optimization process and hence, the different design schemes may end up with different final discretizations, even though the final geometries are the same. The nodal stress distributions along the upper boundaries of the three structures are depicted in Fig. 1(f), where nearly constant nodal stresses are observed for most regions along these boundaries, except for some fluctuations at the supporting and loading regions.

This example clearly demonstrates that compliance minimization, fully stressed and adjoint-based stress
Table 1: Statistics of the optimized cantilever beams in Fig. 1

\begin{tabular}{c|c|c}
\hline Optimization Cases & $\begin{array}{c}\text { Max. elemental } \\
\text { stress } \sigma_{e}^{\max }\end{array}$ & $\begin{array}{c}\text { Compliance } \\
C\end{array}$ \\
\hline Min. Compliance & 11.3 & 3.68 \\
Min. Stress $p=30$ & 10.1 & 3.68 \\
Fully Stressed Design & 11.6 & 3.68 \\
\hline
\end{tabular}

minimization results in the same optimal designs, as long as boundary movement is unconstrained.

\subsection{L-bracket}

Fig. 2(a) illustrates the L-bracket example fixed at the top and with a small, solid, non-design domain where the distributed load is applied as indicated with blue color. The initial guess is shown in Fig. 2(b), which has a radius of 60 at the reentrant corner. It has a maximum elemental stress of 16.3 and occupies $46.875 \%$ of the design domain.

Figs. 2(c-g) show the stress optimized designs obtained for the adjoint approach with different $p$ values. As $p$ increases from 2 to 30 , the maximum elemental stress reduces from 29.8 to 9.0 . Fig. 2(h) shows the FSD result which has a maximum stress of 24.9 (at the re-entrant corner). The corresponding compliance optimized design is shown in Fig. 2(i) which has a maximum stress of 30.5. Fig. 2(j) compares the nodal stress distributions along the upper right structural boundary for all designs. Here it is clear that the FSD as well as the compliance design result in inefficient solutions with huge stress concentrations at the reentrant corner.

To demonstrate that the failure of the FSD strategy to prevent the stress singularity is not due to a bad starting guess or a wrong choice of target stress, the FSD strategy is further tested on the L-bracket case with a good initial guess. As shown in Fig. 3(a), the initial guess (optimized with $p=30$ ) has a maximum nodal stress of $\sigma_{n}^{\max }=9.186$. Figs. $3(\mathrm{~b}-\mathrm{d})$ present the results of FSD for constant volume control and for different predefined target nodal stresses of $\sigma_{n}^{*}=9.187$ and $\sigma_{n}^{*}=12.0$, respectively. The corresponding numbers are listed in Tab. 3. For this example, all the FSD runs naturally start by removing the low-stress region at the lower left part of the initial design and add material to the re-entrant corner region. Unfortunately, such changes result in the opposite effect of what is desired! To further illustrate this point, black and red arrows, indicating the "FSD gradients" and the adjoint $p$-norm gradients (for $p=30$ ), respectively, are added to Figs. $3(\mathrm{a})$ and (b). Clearly, the black "FSD gradients" have 
Table 2: Statistics of the optimized L-brackets in Fig. 2

\begin{tabular}{c|c|c}
\hline Optimization Cases & $\begin{array}{c}\text { Max. elemental } \\
\text { stress } \sigma_{e}^{\max }\end{array}$ & $\begin{array}{c}\text { Compliance } \\
C\end{array}$ \\
\hline$p=2$ & 29.8 & 1.502 \\
$p=4$ & 21.9 & 1.529 \\
$p=8$ & 11.7 & 1.727 \\
$p=12$ & 10.1 & 1.821 \\
$p=30$ & 9.0 & 1.959 \\
Compliance & 30.5 & 1.500 \\
FSD & 24.9 & 1.502 \\
\hline
\end{tabular}

Table 3: Statistics of the optimized L-brackets in Fig. 3

\begin{tabular}{c|c|c}
\hline Optimization Cases & $\begin{array}{c}\text { Max. elemental } \\
\text { stress } \sigma_{e}^{\max }\end{array}$ & Volume ratio \\
\hline Initial design & 9.0 & $46.875 \%$ \\
I: constant volume & 26.8 & $46.875 \%$ \\
II: $\sigma_{n}^{*}=9.187$ & 37.1 & $35.2 \%$ \\
III: $\sigma_{n}^{*}=12.0$ & 44.1 & $30.3 \%$ \\
\hline
\end{tabular}

not only wrong magnitudes but also wrong directions! This leads to the unintended removal of material in the lower left region which in the real optimal structure leads to stress alleviation in the opposing corner region. Also, the FSD strategy is unable to remove material in the high-stress re-entrant region to obtain the wanted smoothed fillet.

The red arrows, corresponding to the gradients of the p-norm objective function in Fig. 3(a) and (b), can also be seen as a "visual KKT check. For the KarushKuhn-Tucker (KKT) conditions to be satisfied the gradient of the objective function minus the Lagrange multiplier times the gradient of the constraint (volume) function must be zero for all non-constrained designs variables. We only indicate the gradient of the objective function by the arrows, however, considering that all elements are close to equilateral and of similar sizes due to the DSC approach used, the gradients of the constraint (volume) function will be nearly constant. Hence, KKT conditions are visually satisfied for the pnorm design (Fig. 3(a)) and certainly not for the FSD design (Fig. 3(b)). At convergence, the infinity norm of the KKT condition is $2.6 \times 10^{-5}$ for the consistently optimized p-norm case and $9.6 \times 10^{-3}$ for the FSD case, again clearly indicating that the FSD approach does not satisfy optimality for geometrically constrained design problems.

Finally, the study for increasing $p$-norm as shown in Figs. 2(c-g) and Tab. 2 shows that large $p$-values are required in order to effectively minimize the maximum stress. Even for $p=8$, the maximum stress is $20 \%$ larger than for $p=30$ and hence high values (cf. $p \geq 10$ ) are recommended.
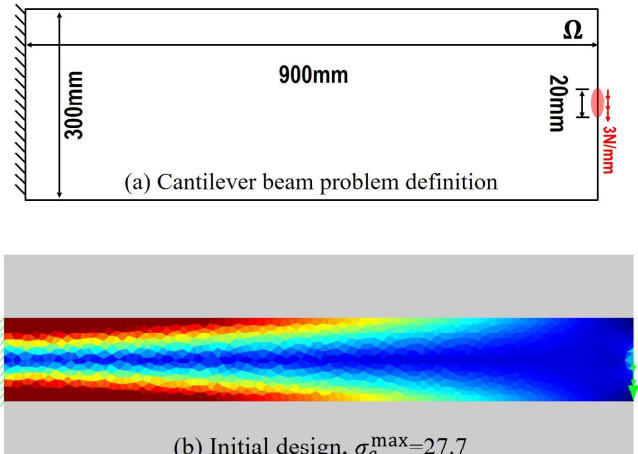

(b) Initial design, $\sigma_{e}^{\max }-27.7$
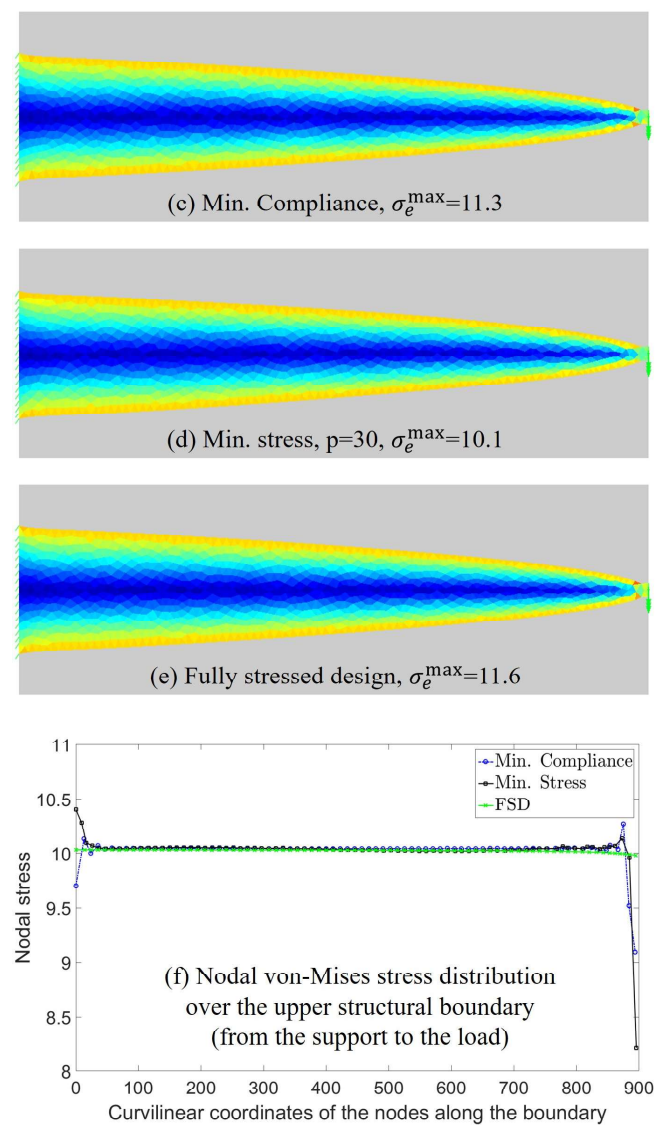

Fig. 1: Shape optimization of a cantilever beam. The color is plotted for the maximum von-Mises stress (at Gaussian points) of each element. Color scale ranges from 0 to 15 (same color for $\sigma_{e} \geq 15$ ).

\section{Conclusions}

By two examples, this short note reminds the readers that the success of so-called Fully Stressed Design (FSD) concepts is limited to geometrically unrestricted design problems and that high $p$-values must be used if using constraint aggregation schemes for treating stress constraints. Both observations should be well-known 


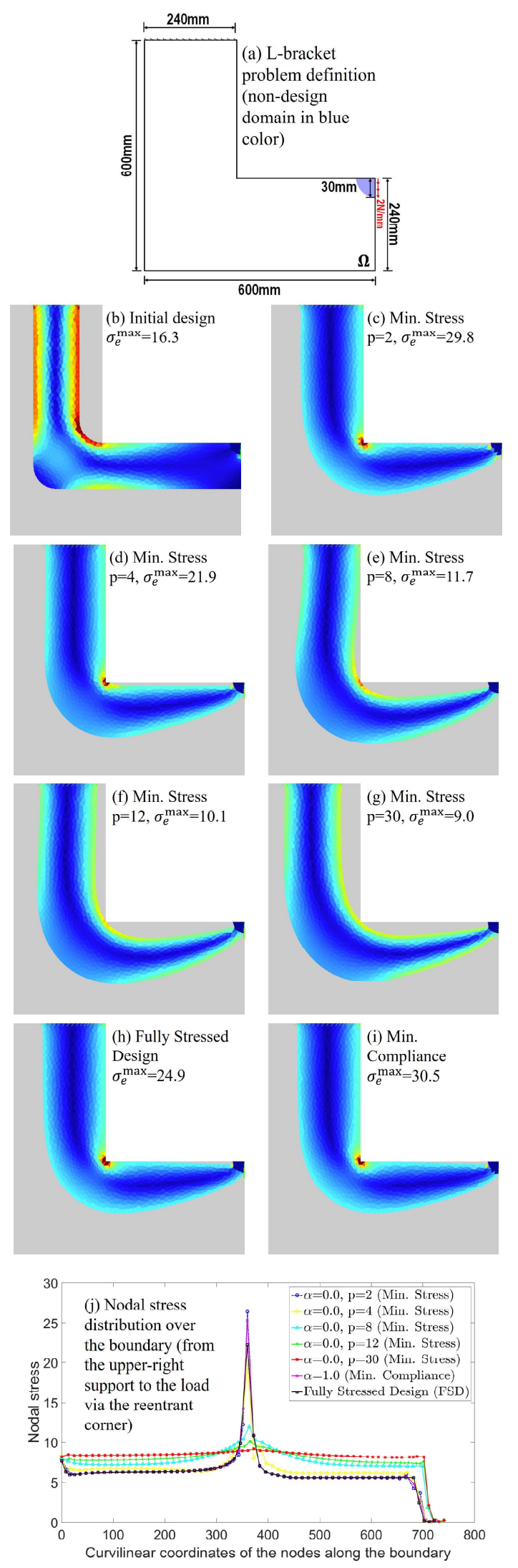

Fig. 2: Shape optimization of a L-bracket. The color scale for stress distribution is the same as in Fig. 1.
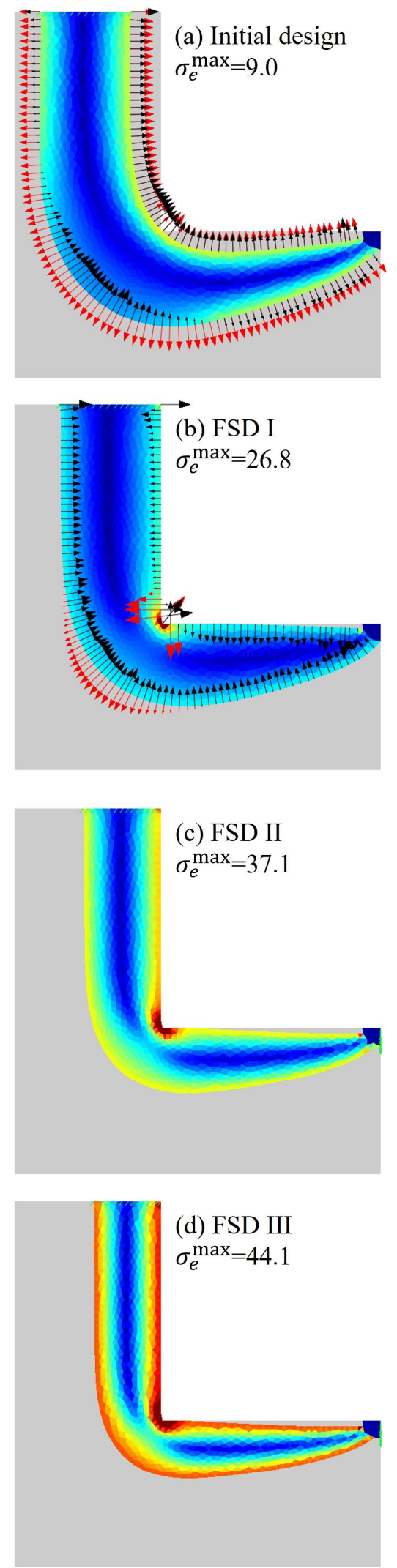

Fig. 3: FSD results with a good initial guess (Fig. 2(f)). The black and red arrows represent the "FSD gradients" and the $p$-norm design gradients (for $p=30$ ). The color scale for the stress plots is the same as in Fig. 1. 
but are nevertheless ignored in large parts of older and newer literature.

Acknowledgements The authors acknowledge the financial support from the research project "Sapere Aude TOpTEn" (Topology Optimization of Thermal ENergy systems) from the Danish Council for Independent Research, grant: DFF4005-00320. 